Originally published as:

Masihi, K.N., Schäfer, H.

Overview of Biologic Response Modifiers in Infectious Disease

(2011) Infectious Disease Clinics of North America, 25 (4), pp. 723-731.

DOI: 10.1016/j.idc.2011.07.002

This is an author manuscript.

The definitive version is available at: http://www.sciencedirect.com/ 


\section{Overview of Biological Response Modifiers in Infectious Disease}

K. Noel Masihi, Ph.D and Hubert Schäfer, Ph.D

Robert Koch Institute, Berlin, Germany

The authors have nothing to disclose.

Keywords: biological response modifiers, immunomodulators, cytokines, infections,

Corresponding author for

proof and reprints:

Coauthor address:

Hubert Schäfer, Ph.D

K. Noel Masihi, Ph.D

Robert Koch Institute

Robert Koch Institute

Nordufer 20

Nordufer 20

D - 13353 Berlin

D - 13353 Berlin

Germany

Germany

(+49 30) 18754-2544

(+49 30) 18654-2602

schaeferh@rki.de

masihik@rki.de 


\section{INTRODUCTION}

Infectious diseases continue to impact human morbidity and mortality. The massive explosions of tourism with an estimated 2 billion passengers each year on commercial airlines and rapid globalization have facilitated the spread of disease-causing pathogens from one continent to another at unprecedented rates. According to the World Health Organization, at least 40 new diseases have emerged over the past two decades, at a rate of one or more per year.

Antimicrobial drugs have been instrumental in saving the lives of millions of people worldwide. The effectiveness of many antibiotics is, however, being steadily eroded by the emergence of drug resistant microorganisms [1] with the WHO declaring that antibiotic resistance is amongst the three greatest challenges to human health. About 25000 patients die each year in the EU from infections with multidrug-resistant bacteria according to the European Medicines Agency causing extra healthcare costs and productivity losses of at least EUR 1.5 billion each year. Crucial drug choices for the treatment of common infections by bacteria, viruses, parasites, and fungi are becoming limited and even nonexistent in some cases [2]. This development has not been paralleled by an effective increase in the discovery of new drugs for most pathogens and the rate of new antimicrobials approvals is steadily dropping. The struggle to control infectious diseases, far from being over, has acquired a new poignancy. Novel concepts acting as adjunct to established therapies are urgently needed. 
The immune system can be manipulated specifically by vaccination or non-specifically by immunomodulation. Biological response modifiers or immunomodulators include both immunostimulatory and immunosuppressive agents. This chapter will concentrate on immunostimulatory agents capable of enhancing host defense mechanisms to provide protection against infections. Other synonymous terms for biological response modifiers include immunostimulants, immunoaugmentors, or immunorestoratives. Their modes of action include augmentation of anti-infectious immunity by the cells of the immune system encompassing lymphocyte subsets, macrophages, dendritic and natural killer cells. Further mechanisms can involve induction or restoration of immune effector functions and tilting the balance towards cytokine pathways germane to protection. A diverse array of recombinant, synthetic, and natural immunomodulatory preparations for prophylaxis and treatment of various infections are available today [3-6].

\section{CYTOKINE IMMUNOMODULATORS}

Cytokines, hormone-like polypeptides possessing pleiotropic properties, are crucial in orchestrating the appropriate immune responses critical for the outcome of an infection. Certain cytokines stimulate the production of other cytokines and interact in synergistic or antagonistic networks enabling manipulation of the host response to enhance overall immunogenicity and direct the nature of the response either toward type 1 or type 2 pathways. In the type 1 response, Th1 cells produce interferon (IFN) $-\gamma$, tumor necrosis factor (TNF) and interleukin (IL)-12 that are required for effective development of cell-mediated immune responses to intracellular microbes. In the type 2 response, Th2 cells produce IL-4, IL- 5 and 
IL-13 that enhance humoral immunity to T-dependent antigens and are necessary for immunity to helminth infections. Recent studies have shown that toll-like receptors (TLR), mainly found on dendritic cells (DCs) and macrophages, are important in transition from innate to adaptive immunity.

In humans, DCs are subdivided into plasmacytoid DCs, which secrete copious amounts of IFN- $\alpha$, and myeloid DCs. Both subtypes of DCs recognize diverse microbial pathogens through specific TLR. Local and systemic effects of cytokines are, thus, intimately involved in the host control of infections. Several recombinant and natural cytokine preparations such as, interferons, and granulocyte colony-stimulating factor are already licensed for use in patients.

\section{INTERFERONS AND COMBINATIONS}

Interferon (IFN) was the first cytokine to be produced by the recombinant DNA technology. There are two classes of interferon, type I and type II. The type I IFN is produced in response to a viral infection and includes IFN- $\alpha$ and IFN- $\beta$. Most of the IFN- $\alpha$ in human is released by plasmacytoid dendritic cells whereas IFN- $\beta$ is produced by fibroblasts and many other cell types. The type II IFN is secreted by activated T cells and NK cells as IFN- $\gamma$ also known as immune interferon.

Interferon-alpha (IFN- $\alpha$ ) is a clinically effective therapy used in a wide range of viral infections besides its application in malignant melanoma, basal cell carcinoma and warts. 
Natural IFN- $\alpha$ obtained from human serum and leukocytes is currently licensed for the treatment of a rare form of cancer, hairy cell leukemia. Recombinant IFN- $\alpha 2 \mathrm{a}$ is licensed for treatment of chronic active hepatitis B and for hepatitis C virus infections.

Standard IFN- $\alpha$ has the drawbacks of a short serum half-life and rapid clearance. To overcome this problem, pegylated forms of IFN (PegIntron or Pegasys) have been developed and tested clinically. The greater polymer size of pegylated IFN acts to reduce glomerular filtration, markedly prolonging its serum half-life (72-96 hours) compared with standard IFN (6-9 hours) [7].

Hepatitis B virus (HBV) infection is widespread throughout the world infecting around 400 million people and killing between 1 and 2.5 million people a year. The virus, transmitted by blood or body fluids, is up to 100 times more infectious than HIV. Overall, 15 to $25 \%$ of HBV carriers die from chronic hepatitis, cirrhosis or hepatocellular carcinoma (HCC) which accounts for up to 90 percent of all liver cancers.

At present, licensed therapies widely used for treating liver disease caused by HBV include IFN- $\alpha$, antiviral drugs lamivudine, adefovir and entecavir [8]. Most of these therapies are limited in the clinic by a low response rate, with only a small subset of patients with hepatitis $\mathrm{B}$ and around $40 \%$ of cases with hepatitis $\mathrm{C}$ being responsive to interferon therapy. 
Several promising studies have shown the effectiveness of pegylated interferon combination therapy with lamivudine or adefovir [9] with a better cost-benefit ratio. Interferon- $\alpha$ is also approved for treating condyloma acuminata caused by human papilloma virus and for Kaposi sarcoma in patients with HIV infection. Besides pegylated interferon, newer drug formulations demonstrated in clinical trials to be active against HBV include tenofovir, telbivudine and clevudine.

Hepatitis $\mathrm{C}$ virus (HCV) is the major ecological agent of post-transfusion and communityacquired non-A, non-B hepatitis infecting over 200 million people worldwide. Combination therapy with pegylated IFN and ribavirin (Pegetron) is the current standard of care for the treatment of chronic HCV infection. Recently, patients with a particular polymorphism of a gene called $I L 28 B$ have been shown to respond more favorably to treatment [10]. The FDA is expected to approve two protease inhibitors, telaprevir and boceprevir, in 2011 as the next generation of anti-HCV treatment.

Interferon- $\beta$ (IFN- $\beta$ ) obtained from human FS-4 fibroblast cell lines is licensed for use in severe uncontrolled virus-mediated diseases in cases of viral encephalitis, herpes zoster and varicella in immunosuppressed patients. A further indication is viral infection of the inner ear with loss of hearing. The standard treatment for multiple sclerosis, a disease without definitively elucidated etiology, is currently IFN- $\beta$. 
Interferon- $\gamma($ IFN- $\gamma)$ is the major mediator of host resistance during the acute and chronic phases of infection and is pivotal in protection against a variety of intracellular pathogens. Multicenter clinical trials have shown that sustained administration of IFN- $\gamma$ to chronic granulomatous disease patients markedly reduced the relative risk of serious infection. IFN- $\gamma$ is licensed as a therapeutic adjunct for use in patients with chronic (septic) granulomatosis for reduction of the frequency of serious infections. Imukin and Actimmune ${ }^{\mathrm{TM}}$ are IFN- $\gamma$ preparations marketed for chronic granulomatous disease, mycobacterial and fungal infections.

The major side effects of all interferon therapies include flu-like syndromes, fever, myalgia, headache and fatigue. Hypotension, granulocytopenia, and thrombocytopenia can also occur. Deleterious effects on central nervous system, particularly at high doses, have been observed.

\section{COLONY-STIMULATING FACTORS}

Granulocyte Colony-Stimulating Factor (G-CSF) preparations such as Filgrastim can significantly enhance neutrophil functions. A high proportion of HIV-infected patients has neutropenia, which considerably increases the risk for bacterial and fungal infections. Filgrastim was granted license extension to cover the treatment of persistent neutropenia at an advanced stage of HIV infection [11]. In one study conducted at 27 European centers on AIDS patients with cytomegalovirus infection, G-CSF (lenograstim) was found to be suitable for the treatment of ganciclovir-induced neutropenia [12]. Recently developed recombinant 
human G-CSF, pegfilgrastim, has been shown to be associated with a lower risk of neutropenia-related hospitalizations [13]

G-CSF and Granulocyte-Macrophage Colony-Stimulating Factor (GM-CSF) are used to reverse leukopenia as adjunctive therapy for HIV-associated infections. The GM-CSF preparation Sargramostim helps overcome defects in neutrophil and macrophage function due to its broad range of effects on lymphocytes, macrophages, neutrophils, and dendritic cells. It enhances the antiretroviral activity of zidovudine and stavudine in macrophages and ameliorates the hematologic side effects of these agents. Multiple deficiencies are involved in the progression of fungal infections in cancer patients with or without neutropenia. Although clinical experience is still limited, G-CSF, GM-CSF, and macrophage colony-stimulating factor show promise as adjuvant therapy for fungal infections.

\section{CHEMOKINES AND CHEMOKINE MODULATORS}

CHEMOKINES have been historically regarded as leukocyte chemoattractants capable of regulating cellular trafficking into inflammatory sites. The sobriquet "chemokine" is abbreviated from chemotactic cytokine. The major classes of chemokines are constituted by the $\mathrm{CXC}$ or $\alpha$ chemokines, the $\mathrm{CC}$ or $\beta$ chemokines, the $\mathrm{C}$ or $\gamma$ chemokines, and the $\mathrm{CX} 3 \mathrm{C}$ chemokines. The nomenclature of the chemokines is based on the spacing of two critical cystine residues near the amino terminus, indicating no $(\mathrm{CC})$ or one $(\mathrm{CXC})$ or three $\mathrm{CX} 3 \mathrm{C})$ aminoacids acids between the first two cysteins near the amino terminus. The chemokine 
family has expanded to 80 ligands including CXCL, CCL, XCL and CX3CL chemokines and chemokine receptors including CXCR, CCR, XCR and CX3CR

Many erudite reviews on human immunodeficiency virus (HIV) /AIDS etiology and manifestation have been published, and these will not be dealt with here. It is noteworthy that currently there are more women worldwide who have been infected with HIV than males and females account for nearly half of over 40 million people living with HIV [14]. Women are more vulnerable to HIV infection than men, and their increased susceptibility has been linked to the use of hormonal contraceptives and sexually transmitted diseases. Also, susceptibility to HIV varies throughout a women's reproductive life; adolescent girls appear to be most vulnerable to HIV due to high-risk sexual behavior and a not fully mature reproductive system. In addition, recent studies have indicated increases in the risk of acquiring HIV during pregnancy and during the early postpartum period, part of which could be attributed to higher levels of progesterone. Successful control of the HIV pandemic requires continuing focus on gender. Some of the fundamental issues of HIV transmission have been riddled with problems as it is neither feasible to obtain nor examine relevant cells and tissue at the precise time of HIV acquisition.

The binding of the HIV envelope glycoprotein gp120 to CD4 and appropriate chemokine receptors triggers conformational changes facilitating the fusion of the viral and host cell membranes. Chemokine receptors - mainly CCR5 and CXCR4 - have been discovered to be necessary as coreceptors for HIV entry [15]. HIV found in the vaginal and rectal mucosa is mainly CCR5-dependent. Macrophage-tropic (R5) HIV variants predominantly make use of 
the CCR5 coreceptors. The T cell-tropic (X4) and dual-tropic (R5X4) HIV strains, generally associated with the clinical manifestations of AIDS, emerge after a latency of several years, although pathogenesis of the central nervous system and related symptoms are normally associated with M-tropic (R5) HIV strains. X4 HIV infection augments the expression of chemokines such as MIP-1 $\alpha$ (macrophage inflammatory protein) and RANTES (regulated upon activation normal T cell expressed and secreted) [16].

HIV infection can be inhibited by chemokines and chemokine-related molecules that are ligands for receptors that function as coreceptors. Chemokine receptors thus represent important targets for intervention in HIV and search for molecules that have a therapeutic potential as inhibitors of these receptors has been intense [17]. Intervention strategies based on chemokine antagonists that could be useful for the therapy of HIV include receptor-ligand interaction, prevention of the chemokine-glycosaminoglycan interaction, interfering with the signaling pathways that are induced upon receptor activation, and modification of receptor pathways [18].

\section{TARGETING CCR5 AND CXCR4}

The chemokine receptors CXCR4 and CCR5 are the main coreceptors used respectively by the T-cell-tropic (CXCR4-using, X4) and macrophage-tropic (CCR5-using, R5) HIV for cell entry. Several compounds targeting CXCR4 and CCR5 have been advocated recently. The idea for the drug class came from the observation that presence of mutated CCR5 can confer resistance to HIV infection, even after exposure to numerous high-risk sexual partners. Only around 2 percent of Caucasians carry such a mutation. 
Modified chemokines with antiviral activity, several low-molecular weight CXCR4 and CCR5 antagonistic compounds, have been described. Maraviroc developed by Pfizer as a CCR5 antagonist, was licensed in 2007 by the FDA for use in HIV treatment-experienced patients harboring only R5 viruses. Another chemokine (CCR5) inhibitor, Aplaviroc or GSK 873140, was developed by GlaxoSmithKline, but studies were discontinued due to liver toxicity concerns. Other companies working on CCR5 antagonists include Pfizer Inc and Schering-Plough Corp.

Plerixafor (AMD3100, Mozobil), a bicyclam compound, targets CXCR4 and has a potent anti-HIV activity against T-tropic viruses. It is used in combination with G-CSF to enhance mobilization of hematopoietic stem cells in transplantation patients with non-Hodgkin's lymphoma or multiple myeloma and has a good safety profile. It has marketing authorization in 33 countries worldwide including the EU and USA and is commercially available in 12 countries. A derivative AMD3451, pyridinylmethyl monocyclam, has been synthesized and reported to show dual CCR5/CXCR4 antagonistic activity against both X4 and R5 HIV strains [19].

Most existing HIV drugs work inside the body's immune cells, after the virus has infected and can cause anemia, diarrhea and nerve pain. New drugs could provide an important treatment option for people with HIV/AIDS, by offering a different mode of action and an improved toxicity profile. Both CXCR4 and CCR5 chemokine receptor inhibitors may be needed in 
combination and even in combinations with antiviral drugs that also target other aspects of the HIV replication cycle to obtain optimum antiviral therapeutic effects.

\section{CYTOKINE INHIBITORS}

Several strategies exist for responding to infection. One mechanism by which the host attempts to restrain the infection is through the up-regulation of cytokines. Some cytokines, such as IL-1, IL-6, IL-8, IL-18, and TNF, counteract the challenge by worsening the disease in an effort to rid the host of infection. Overproduction of proinflammatory cytokines is believed to underlie the progression of many inflammatory diseases including rheumatoid arthritis, Crohn's disease and endotoxin shock. Many infectious diseases, including HIV, influenza H5N1 and malaria can induce deleterious overproduction of proinflammatory cytokines.

Intense interest has been generated in developing agents that can block the activity of such cytokines. Inhibition of TNF activity has been singularly successful in the treatment of autoimmune diseases. Monoclonal antibodies including adalimumab (Humira; Abbott), etanercept (Enbrel; Amgen/Wyeth), infliximab (Remicade; Centocor), and Certolizumab pegol are used in the treatment of rheumatoid arthritis. An interleukin-1 (IL-1) receptor antagonist, Kineret, has also been licensed. Some of these monoclonal antibody products have shown promise for applications in disease management in patients with HIV/AIDS [20-21] 
Another potent TNF inhibitor, thalidomide, has been used in trials in HIV patients [22]. More recently, thalidomide and its immunomodulatory imide analogs such as Actimid, Lenalidomide, Pomalidomide and Revlimid, have been used as anti-inflammatory drugs.

Pentoxifylline, a methylxanthine usually used in the treatment of peripheral arterial circulatory disorders, has been shown to inhibit TNF synthesis. Pentoxifylline has been used in HIV for improving certain functions [23]. Currently, clinical trials are ongoing with phosphodiesterase inhibitors and small-molecule inhibitors of TNF-converting enzyme that specifically interrupt the signaling pathways of TNF.

TNF plays an important role in defense against bacterial and viral infections. Consequently, many patients on anti-TNF drug therapy have shown severe infections such as tuberculosis [24]. A variety of other opportunistic fungal infections by Candidia, Aspergillus Pneumocystis, Coccidioides, Histoplasma, have also been reported in patients treated with anti-TNF monoclonals [25].

Sepsis is one of the most common complications in surgical patients and one of the leading causes of mortality in intensive care units. Sepsis can be caused by infection with Gramnegative bacteria, Gram-positive bacteria, fungi, or viruses. Sepsis may, however, also occur in the absence of detectable bacterial invasion. In such cases, microbial toxins, particularly Gram-negative bacterial endotoxin, and endogenous cytokine release have been implicated as initiators and mediators. Septic shock represents the most severe form of host response to infection. Despite recent progress in antibiotics and critical care therapy, sepsis is still associated with a high mortality rate $(\sim 40-50 \%)$ and remains the tenth leading cause of death in western countries. A number of therapies delaying the onset, and/or reducing the effects of 
proinflammatory cytokines induced during sepsis are under development. TAK-242 (Resatorvid), a small molecule antagonist of Toll-like receptor (TLR) 4 signaling, reduces lipopolysaccharide (LPS)-induced production of proinflammatory IL-1, IL-6 and TNF-and is currently undergoing phase III evaluation [26]. Another compound, E5564 (Eritoran), is a synthetic molecule derived from the nonpathogenic Rhodobacter sphaeroides and was observed to reduce TNF and IL-6 levels after LPS administration [48]. Eritoran is under evaluation in a phase III study in patients with onset of severe sepsis.

\section{SYNTHETIC IMMUNOMODULATORS}

Microbial pathogens possess a variety of evolutionarily conserved structural motifs known as pathogen-associated molecular patterns (PAMPs). These PAMPS are recognized by a family of specific TLRs. Around thirteen TLRs exhibiting distinct ligand specificities have been identified in humans. TLR2 recognizes bacterial peptidoglycan and lipopeptide, TLR3 recognizes double-stranded RNA, TLR4 binds to lipopolysaccharide, TLR5 binds to flagellin that is part of the flagellum that propels many kinds of bacteria. TLR7 and TLR8 recognize imidazoquinoline compounds and single-stranded RNA from viruses, whereasTLR9 binds to unmethylated CpG DNA motifs frequently found in the genome of bacteria and viruses.

In humans, TLR7 and TLR9 are expressed on the plasmacytoid dendritic cells (PDC) which can rapidly synthesize large amounts IFN- $\alpha$ and IFN- $\beta$ in response to viral infection. Interestingly, TLR7 recognizes synthetic immunomodulators such as Imiquimod, a fully synthetic immune response enhancing imidazoquinoline amine [28], that are used against viral infections. Imiquimod is marketed as Aldara ${ }^{\mathrm{TM}}$ for genital warts caused by human papillomavirus (HPV) subtypes number 6 and 11, but is widely used for basal cell carcinoma, 
actinic keratosis and molluscum contagiosum. It has shown promise in lentigo maligna and cutaneous metastases of malignant melanoma.

\section{Emerging therapies with synthetic immunomodulators}

\section{Antimicrobial Peptides}

Antimicrobial peptides (AMPs), also known as host defense peptides (HDPs), are present mainly in phagocytic cells of the immune system and are rapidly mobilized. AMPs demonstrate potent antimicrobial activity and can kill a wide array of Gram-positive and Gram-negative bacteria, enveloped viruses, fungi and parasites through disruption of microbial membranes. Of clinical significance is the ability of AMPs to kill multidrugresistant microorganisms [29]. Two AMPs have been shown in Phase III clinical trials to be effective. Pexiganan, has been developed for topical treatment of patients with mild diabetic foot infection and can cure approximately $90 \%$ of the patients. The other AMP, Omiganan, has shown efficacy in preventing catheter-related infections.

\section{Defensins}

Defensins are polypeptides of the innate immune system produced in response to microbial infection in humans and animals. Recent reports have highlighted the anti-HIV activities of defensins, whose structure and charge resemble portions of the HIV-1 transmembrane envelope glycoprotein gp41. CD8 T cells from long-term non-progressors with HIV infection 
were found to secrete a cluster of proteins identified as $\alpha$-defensin 1,2, and 3. A study of seronegative women who were exposed constantly to HIV-1 demonstrated that their CD $8^{+}$ cells exhibit extensive $\alpha$-defensin production at both peripheral and mucosal levels. The $\alpha$ defensin expression level in these seronegative women was 10-fold higher than that of control subjects [30]. Likewise, overexpression of $\alpha$-defensins in breast milk results in a low rate of HIV-1 transmission from mother to infant [31].

HIV induces $\beta$-defensin- 2 and 3 in human oral epithelial cells which exhibit strong anti-HIV activity due to the direct antiviral effect or competition for the chemokine receptors that HIV uses to enter the cell [32]. Mother-to-child transmission of HIV is the main source of pediatric AIDS. There is a significant relationship between genetic variants of $\beta$-defensin- 1 gene, viral load, and mother-to-child transmission of HIV. In mothers, the -52GG genotype is associated with low levels of HIV plasma viremia and a lower risk of maternal HIV transmission [33].

Defensins are known to exhibit inhibitory activity against several viruses. Alpha-defensins promote uptake of influenza virus by neutrophils and human defensins 5 and 6 are effective in neutralizing influenza virus [34]. The expression of murine $\beta$-defensin was enhanced in influenza-infected lungs, trachea and nasal mucosa [35] and treatment of cell cultures with human neutrophil peptides -soon after infection - resulted in marked inhibition of influenza virus replication and viral protein synthesis [36]. BK virus is a polyomavirus that establishes a lifelong persistence in most humans. Studies have shown that human alpha-defensins can inhibit BKV infection [37], inhibit adenovirus infection [38], and $\alpha$-defensins and human $\beta$ defensin inhibited herpes simplex virus infection [39]. 


\section{Synopsis}

The conventional treatment of infectious agents is increasingly encountering antimicrobial resistance. This has led to an intense search for novel treatment modalities for infectious diseases including therapeutic use of biologically manufactured cytokines and cytokine inhibitors. The appeal of biological response modifiers is inherent in modes of action that are distinctly different from the direct microbicidal action of established antibiotic, antiviral, antifungal and antiparasitic drugs. Elucidation of mechanisms underlying the inhibitory activity of chemokines has been instrumental in the rational design of anti-HIV chemokine drugs such as Maraviroc and other analogs. The immune-based therapies in combination with antimicrobial drugs, such as interferons and ribavirin for viral hepatitis have attracted much attention. Recognition of toll-like receptors by synthetic immunomodulators such as Imiquimod are used for certain viral infections. New methodologies have the potential to identify novel targets and foster the development of individually tailored immunomodulatory drug treatments. 


\section{References}

[1] Gottlieb T, Nimmo GR. Antibiotic resistance is an emerging threat to public health: an urgent call to action at the Antimicrobial Resistance Summit. Med J Aust 2011; 21;194:281-3.

[2] Amyes SGB. The rise in bacterial resistance is partly because there have been no new classes of antibiotics since the 1960s. Br Med J 2000; 320: 199-200.

[3] Hengel H, Masihi KN. Combinatorial immunotherapies for infectious diseases. Int Immunopharmacol 2003; 3: 1-9.

[4] Kayser O, Masihi KN, Kiderlen AF. Natural products and synthetic compounds as immunomodulators. Expert Rev Anti-infect Ther 2003; 1: 319-35.

[5] Masihi KN. Immunomodulatory agents for prophylaxis and therapy of infections. International Journal of Antimicrobial Agents 2000; 14: 181-91.

[6] Masihi KN. Fighting infection using Immunomodulatory agents. Expert Opin Biol Ther 2001; 1: 641-53.

[7] Luxon BA, Grace M, Brassard D, Bordens R. Pegylated interferons for the treatment of chronic hepatitis C infection. Clinical Therapeutics 2002; 24: 1363-83.

[8] Brunetto MR, Lok AS. New approaches to optimize treatment responses in chronic hepatitis B. Antivir Ther. 2010;15:61-8.

[9] Villa E, Lei B, Taliani G,et al. Pretreatment with pegylated interferon prevents emergence of lamivudine mutants in lamivudine-naive patients: a pilot study. Antivir Ther. 2009;14:1081-7. 
[10] Wapner J. Gene variants affect Hepatitis C treatment, but link is elusive. Science 2010; 330: 579 .

[11] Welch W, Foote M. The use of Filgrastim in AIDS-related neutropenia. J Hematother Stem Cell Res 1999; Suppl 1:S9-16.

[12] Dubreuil-Lemaire ML, Gori A, Vittecoq D, et al. Lenograstim for the treatment of neutropenia in patients receiving ganciclovir for cytomegalovirus infection: a randomised, placebo-controlled trial in AIDS patients. Eur J Haematol 2000; 65: 337-43.

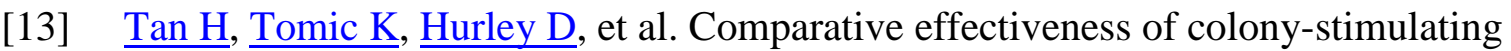
factors for febrile neutropenia: a retrospective study. Curr Med Res Opin 2011;27:79-86.

[14] Quinn TC, Overbaugh J. HIV/AIDS in women: an expanding epidemic. Science 2005; 308: $1582-83$

[15] Dragic T. An overview of the determinants of CCR5 and CXCR4 co-receptor function. J Gen Virol 2001; 82: 1807-14.

[16] Wetzel MA, Steele AD, Henderson EE, et al. The effect of X4 and R5 HIV-1 on C, C$\mathrm{C}$, and $\mathrm{C}-\mathrm{X}-\mathrm{C}$ chemokines during the early stages of infection in human PBMCs. Virology $2002 ; 292: 6-15$

[17] Lusso P. HIV and chemokines: implications for therapy and vaccine. Vaccine 2002; 20: $1964-67$.

[18] Proudfoot AE, Power CA, Rommel C, et al. Strategies for chemokine antagonists as therapeutics. Semin Immunol 2003; 15: 57-65.

[19] Princen K, Hatse S, Vermeire K, Aquaro S, De Clercq E, Gerlach LO et al. Inhibition of Human Immunodeficiency Virus Replication by a Dual CCR5/CXCR4 Antagonist. J Virol 2004; 78: 12996-13006. 
[20] Ting PT, Koo JY. Use of etanercept in human immunodeficiency virus (HIV) and acquired immunodeficiency syndrome (AIDS) patients. Int J Dermatol 2006; 45: 689-92. [21] Cepeda EJ, Williams FM, Ishimori ML, et al. The use of anti-tumour necrosis factor therapy in HIV-positive individuals with rheumatic disease. Ann Rheum Dis. 2008 May;67:710-2.

[22] Johnson L, Jarvis JN, Wilkins EG, Hay PE. Thalidomide treatment for refractory HIVassociated colitis: a case series. Clin Infect Dis 2008; 47: 133-6.

[23] Gupta SK, Johnson RM, Mather KJ et al. Anti-inflammatory treatment with pentoxifylline improves HIV-related endothelial dysfunction: a pilot study. AIDS. 2010; 24(9):1377-80

[24] Strangfeld A, Listing J. Infection and musculoskeletal conditions: Bacterial and opportunistic infections during anti-TNF therapy. Best Pract Res Clin Rheumatol 2006; 20: $1181-95$

[25] Tsiodras S, Samonis G, Boumpas DT, Kontoyiannis DP. Fungal infections complicating tumor necrosis factor alpha blockade therapy. Mayo Clin Proc 2008; 83: 181-94. [26] Rice TW, Wheeler AP, Bernard GR et al. A randomized, double-blind, placebocontrolled trial of TAK-242 for the treatment of severe sepsis. Crit Care Med. 2010;38:168594.

[27] Tidswell M, Tillis W, Larosa SP et al. Phase 2 trial of eritoran tetrasodium (E5564), a toll-like receptor 4 antagonist, in patients with severe sepsis. Eritoran Sepsis Study Group. Crit Care Med. 2010;38:72-83.

[28] Stanley MA. Imiquimod and the imidazoquinolones: mechanism of action and therapeutic potential. Clin Exp Dermatol 2002; 27: 571-77.

[29] Kruse T, Kristensen HH. Using antimicrobial host defense peptides as anti-infective and immunomodulatory agents. Expert Rev Anti Infect Ther 2008; 6: 887-95. 
[30] Trabattoni D, Caputo SL, Maffeis G, Vichi F, Biasin M, Pierotti P et al. Human alpha defensin in HIV-exposed but uninfected individuals. J Acquir Immune Defic Syndr 2004; 35: $455-63$

[31] Kuhn L, Trabattoni D, Kankasa C, Semrau K, Kasonde P, Lissoni F et al. Alphadefensins in the prevention of HIV transmission among breastfed infants. J Acquir Immune Defic Syndr 2005; 39: 138-42.

[32] Garzino-Demo A. Chemokines and defensins as HIV suppressive factors: an evolving story. Curr Pharm Des 2007; 13: 163-72.

[33] Ricci E, Malacrida S, Zanchetta M, Montagna M, Giaquinto C, De RA. Role of betadefensin-1 polymorphisms in mother-to-child transmission of HIV-1. J Acquir Immune Defic Syndr 2009; 51: 13-19.

[34] Doss M, White MR, Tecle T, Gantz D, Crouch EC, Jung G et al. Interactions of alpha, beta-, and theta-defensins with influenza A virus and surfactant protein D. J Immunol 2009; 182: $7878-87$.

[35] Chong KT, Thangavel RR, Tang X. Enhanced expression of murine beta-defensins (MBD-1, -2,- 3, and -4) in upper and lower airway mucosa of influenza virus infected mice. Virology 2008; 380: 136-43.

[36] Salvatore M, Garcia-Sastre A, Ruchala P, Lehrer RI, Chang T, Klotman ME. alphaDefensin inhibits influenza virus replication by cell-mediated mechanism(s). J Infect Dis 2007; 196: 835-43.

[37] Dugan AS, Maginnis MS, Jordan JA, Gasparovic ML, Manley K, Page R et al. Human alpha-defensins inhibit BK virus infection by aggregating virions and blocking binding to host cells. J Biol Chem 2008; 283: 31125-32.

[38] Smith JG, Nemerow GR. Mechanism of adenovirus neutralization by Human alphadefensins. Cell Host Microbe 2008; 3: 11-19. 
[39] Hazrati E, Galen B, Lu W, Wang W, Ouyang Y, Keller MJ et al. Human alpha- and beta-defensins block multiple steps in herpes simplex virus infection. J Immunol 2006; 177: 8658-66. 